\title{
A New Form of Inherited Red-Blindness Identified in Zebrafish
}

\author{
Susan E. Brockerhoff, ${ }^{1}$ James B. Hurley, ${ }^{2}$ Gregory A. Niemi, ${ }^{2}$ and John E. Dowling ${ }^{1}$ \\ ${ }^{1}$ Department of Molecular and Cellular Biology, The Biological Laboratories, Harvard University, Cambridge, \\ Massachusetts 02138, and '2Department of Biochemistry and Howard Hughes Medical Institute, University of \\ Washington, Seattle, Washington 98195
}

\begin{abstract}
A red-blind zebrafish mutant, partial optokinetic response $b$ (pob), has been isolated by measuring eye movements of larvae in a three-generation screen for recessive mutations affecting the visual system. pob larvae exhibit eye movements in response to rotating black and white stripes illuminated with white light, but they do not move their eyes when the stripes are illuminated with red light. Physiological, immunohistochemical, and in situ hybridization analyses of pob retinas showed a selective loss of red-sensitive cones at 5 days postfertilization (dpf). At $3 \mathrm{dpf}$, cells expressing red
\end{abstract}

opsin are present, suggesting that red-sensitive cones form initially but then disappear rapidly, whereas other photoreceptors remain. Linkage analysis indicated that the mutation identified in the pob mutant is not at the red opsin locus. Because red opsin is the only known molecule unique to red cones, these data suggest that a novel gene is required for the maintenance or function of red cones.

Key words: zebrafish; retina; red opsin; vision; behavior; mutant
We have initiated a genetic analysis of zebrafish (Danio rerio) so that we can use its sophisticated visual system to identify genes required for normal vertebrate visual responses. Zebrafish are tetrachromatic; in addition to rods, their retinas contain four types of cones that absorb light maximally in the red $(570 \mathrm{~nm})$, green $(480 \mathrm{~nm})$, blue $(415 \mathrm{~nm})$, and UV $(362 \mathrm{~nm})$ regions of the spectrum (Robinson et al., 1993). Early in development, zebrafish vision is primarily photopic, and it is not until 12 days postfertilization (dqf) that scotopic visual behavior is measurable (Clark, 1981; Branchek, 1984). In adults, the cones are arranged in a row mosaic (Branchek and Bremiller, 1984; Robinson et al., 1993); red- and green-sensitive cones alternate in sequence and are separated by either a single blue cone (always adjacent to a red-sensitive cone) or a single UV cone (always adjacent to a green-sensitive cone) (Robinson et al., 1993). Red opsin expression begins at $\sim 52 \mathrm{hr}$ postfertilization, followed by blue and then by UV opsin (Raymond et al., 1995) (E. Schmitt and J. Dowling, unpublished observation). By $\sim 3$ dpf, zebrafish have opsinexpressing cones distributed throughout the photoreceptor layer of the retina.

We described earlier how a rapid behavioral assay based on the optokinetic response (OKR) can be used to isolate zebrafish larvae with vision defects but with no obvious external morphological abnormalities (Brockerhoff et al., 1995). Zebrafish larvae, by 5 dpf, reliably display smooth pursuit and saccade eye movements in response to illuminated rotating stripes (see http://weber.u.

\footnotetext{
Received Dec. 30, 1996; revised Feb. 7, 1997; accepted March 12, 1997.

This work was supported by National Institutes of Health Grants EY06762-01 (S.E.B.), EY00811, EY00824 (J.E.D.), and EY06641 (J.B.H.), and by the Howard Hughes Medical Institute (J.B.H.). We thank Glenda Froelick for cutting frozen sections, Anthony Scotti for assistance with the linkage analysis, Bill McCarthy, Amy Chin, Nathaniel Hanson, Snorri Gunnarsson, and Jim Fadool for assistance with the screen and for maintaining the Harvard fish facility. We also thank Drs. Abner Lall, Alan Adolph, and Haohua Qian for advice and assistance with ERG recordings and for providing equipment for ERG analyses.

Correspondence should be addressed to Susan E. Brockerhoff at her present address: University of Washington, Department of Biochemistry, Box 357350, Seattle, WA 98195.

Copyright (C) 1997 Society for Neuroscience $0270-6474 / 97 / 174236-07 \$ 05.00 / 0$
}

washington.edu/ jbhurley/Movies.html for a demonstration of the OKR). As a secondary assay, we record the electroretinogram (ERG) from fish that lack an OKR to determine whether the defect resides in the outer retina. Using these two assays we have been able to isolate visual mutants with subtle retinal defects.

In this study we conducted a similar screen using the same behavioral assay; however, we specifically searched for recessive mutations affecting red color pathways to isolate mutations limited to subsets of cells. We describe here a red-blind zebrafish mutant, partial optokinetic response $b$ (pob), in which red-sensitive cones specifically degenerate because of a recessive mutation in a gene other than the red opsin gene. This study shows that a gene in addition to red opsin is required for the function or stability of red cones in zebrafish and thus may define a novel molecule specific for red cones.

\section{MATERIALS AND METHODS}

Mutagenesis and genetics. Zebrafish mutagenesis was performed at the Biological Laboratories at Harvard by methods described previously (Mullins et al., 1994; Solnica-Krezel et al., 1994). Approximately $1 \mathrm{gm}$ of $N$-ethyl- $N$-nitrosourea (ENU) (Sigma, St. Louis, MO) was dissolved in 10 $\mathrm{mm}$ acetic acid to a final concentration of $\sim 100 \mathrm{~mm}$. The precise concentration was determined by measuring the optical density of the solution at $238 \mathrm{~nm}$ (extinction coefficient at $238 \mathrm{~nm}$ is $5830 \mathrm{~m}^{-1} \mathrm{~cm}^{-1}$ at $\mathrm{pH}$ 6.0 ), and the ENU solution was diluted to $3 \mathrm{~mm}$ in fish water (2 gm of Instant Ocean/gallon distilled water) containing $5 \mathrm{~mm}$ sodium phosphate, $\mathrm{pH}$ 6.6. Thirty, 3- to 8-month old $\mathrm{AB}$ strain zebrafish males (Westerfield, 1995) were placed into the $3 \mathrm{~mm}$ ENU solution $(\sim 15$ males/1.5 liters of ENU solution) and left in the hood at $21-22^{\circ} \mathrm{C}$ for $1 \mathrm{hr}$ and then transferred to fresh water ( $\sim$ liters) and left overnight in the hood. Over the course of several hours, the temperature was raised to $28^{\circ} \mathrm{C}$. The following day, fish were fed twice and received two $100 \%$ water changes while they were still in the hood. That evening the mutagenized males were transferred to two 10 gallon fish tanks. This mutagenesis procedure was repeated three times at weekly intervals. Generation of F3 larvae for screening was performed as described previously (Brockerhoff et al., 1995).

One ENU-induced allele of pob ( $p o b^{a l}$ ) was identified from one pair of G0 heterozygotes (F2 fish in the original screen). These fish were outcrossed with wild-type AB strain fish to generate F1 fish. Several pairs of $\mathrm{F} 1$ fish heterozygous for the $p o b$ mutation were identified by screening 


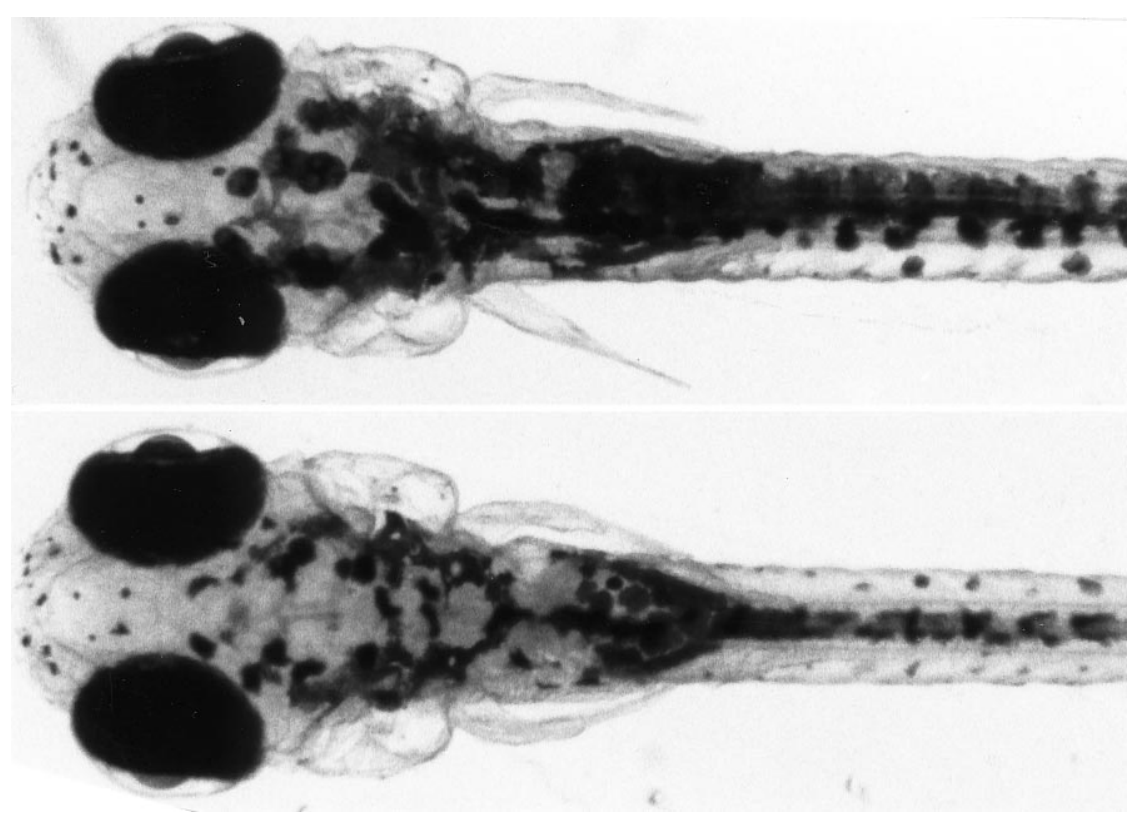

Figure 1. Dorsal view of a 6 dpf pob larva (bottom) and a normal sibling responder larva (top). Anterior is left and posterior is right. Note the slightly smaller melanophore size in the pob mutant. Otherwise, $p o b$ and the sibling responder larvae appear the same. the OKR of progeny from pairwise crosses. Heterozygote F1 fish were again outcrossed with the $\mathrm{AB}$ strain and several pairs of $\mathrm{F} 2$ fish heterozygous for the $p o b$ mutation were identified. $p o b$ mutants isolated from crosses between either F1 or F2 heterozygotes were used for the studies reported here. Heterozygotes identified from an outcross between $A B$ and Ekwill strain zebrafish (Ekwill Fish Farms, Gibsonton, FL) were also isolated. These fish continued to transmit the pob mutation in Mendelian fashion consistent with a recessive mutation at a single locus.

Mutant screening. Screening for behavioral mutants with abnormal OKRs was performed as described previously (Brockerhoff et al., 1995). Larvae were placed in a $35 \mathrm{~mm}$ petri dish containing $\sim 4 \%$ methyl cellulose to partially immobilize them. The dish was then placed in the center of a microscope stage on which a circular drum was mounted. The light source consisted of a red bulb $(600-620 \mathrm{~nm})$ that had a piece of red acetate taped in front of it. The light was reflected off a piece of white paper above the drum down into the drum. The intensity of red light was $5 \mu \mathrm{W} / \mathrm{cm}^{2}$ and the intensity of white light (room light-incandescent bulb) was $3 \mu \mathrm{W} / \mathrm{cm}^{2}$. The drum had $10^{\circ}$ black and white vertical stripes on the inside and was turned at $11 \mathrm{rpm}$ by a belt attached to an adjacent motor. For each larva, the drum was rotated in two directions, and the eye movements were analyzed by watching the larva on a TV monitor attached to the OKR microscope. The stage was illuminated from below with $750 \mathrm{~nm}$ light that was detected by a nearly infrared video camera. Larvae between 3 and $10 \mathrm{dpf}$ did not move their eyes in response to rotating stripes illuminated with $750 \mathrm{~nm}$ light. A response was considered positive if a single smooth pursuit and saccade eye movement in the proper direction was observed after drum rotation was started in each direction. A larva was considered abnormal if it showed no eye movements at all. On average, 1-2 min were required to analyze each larva, including time spent arranging it.

Electroretinography. Electroretinograms were recorded using 10-30 $\mu \mathrm{m}$ tip diameter suction electrodes positioned on the corneas of 5 and $6 \mathrm{dpf}$ pob mutant larvae and their normal siblings, as described previously (Brockerhoff et al., 1995). Spectral sensitivity measurements were made using interference filters and neutral density filters with a $20 \mu \mathrm{V}$ b-wave above baseline as the criterion for a threshold response. For the data shown in Figure $2 c, n=2-5$ at each wavelength for normal larvae and $n=$ 4-8 at each wavelength for $p o b$ mutants. Photon fluxes at each wavelength were determined using a radiometric detector. For analyses of ERG kinetics, $10 \mathrm{msec}$ flashes of white light ( $\sim 30$ lux $)$ approximately one order of magnitude above threshold were used.

Nuclear staining. Ten micrometer cryosections were incubated with a 1:10,000 dilution of 4', 6-diamidino-2-phenylindole (DAPI; stock 2.5 $\mu \mathrm{g} / \mathrm{ml}$ ) in PBS for $5 \mathrm{~min}$ at room temperature and then rinsed three times for 5 min with PBS. Nuclei in the outer nuclear layer were counted in sections that included or were near to the optic nerve. The nuclei from at least three larvae were counted from each time point from which the mean $\pm \mathrm{SD}$ was calculated.
In situ hybridization of normal and pob larvae. Syntheses of digoxygenin-labeled RNA probes and whole-mount in situ hybridization experiments were carried out as described previously, with the following modifications (Oxtoby and Jowett, 1993). Larvae at $5 \mathrm{dpf}$ were not treated with 1-phenyl-2-thiourea (PTU) because PTU treatment made it difficult to distinguish between mutant and nonmutant larvae by the OKR assay. Wild-type PTU-treated larvae did not show consistent OKR responses. Because the larvae were pigmented, detection of photoreceptor staining in whole-mount larvae was difficult, so the retinas were sectioned. Larvae before $4 \mathrm{dpf}$ were treated with $0.0003 \%$ PTU beginning at $24 \mathrm{dpf}$ to make examination of retinal staining possible in retinal whole mounts. The hybridization temperature used for all probes was $65^{\circ} \mathrm{C}$. The larvae were incubated with a 1:2000 dilution of the anti-digoxygenin antibody overnight at $4^{\circ} \mathrm{C}$ and stained for $2 \mathrm{hr}$ at room temperature with 4-nitro blue tetrazolium chloride and 5-bromo-4 chloro-3 indolyl-phosphate (Boehringer Mannheim, Indianapolis, IN). After staining, embryos were dehydrated in a graded series of ethanol solutions and infiltrated with eponaraldite (Schmitt and Dowling, 1994). Larval eyes were sectioned transversely at 5-7 $\mu \mathrm{m}$. Approximately $3 \mathrm{ng} / \mu \mathrm{l}$ probe was used in each experiment, and the same quantity of probe was used in mutant and sibling nonmutant samples. Sense probes were used in at least one experiment to confirm that the staining observed with the antisense probes was specific. Furthermore, mutant and sibling nonmutant embryos were always stained simultaneously. All five normal siblings that were examined from two crosses between pob heterozygotes showed robust red opsin mRNA expression, whereas all seven pob larvae examined from the same two crosses did not. For quantitation of photoreceptor number, stained photoreceptors were counted in tangential sections that either contained the optic nerve or were very near the optic nerve. At least two sections from a minimum of two animals were counted for each probe, and the mean \pm SD was calculated. Partial cDNA clones for zebrafish blue and red opsin were prepared in our laboratory (Reece et al., 1993). The cDNA encoding the goldfish UV opsin gene (Stenkamp et al., 1996), kindly provided by Dr. Pamela Raymond (University of Michigan), was used to identify UV-sensitive cones in zebrafish (Raymond et al., 1996).

Immunocytochemistry. pob larvae and normal sibling responder larvae were fixed for $2 \mathrm{hr}$ at room temperature or overnight at $4^{\circ} \mathrm{C}$ in PBS containing $4 \%$ paraformaldehyde and $3 \%$ sucrose. The embedding of larvae in O.C.T. (Tissue-Tek, Elkhart, IN) and the subsequent staining of sections was performed as described (Westerfield, 1995). Section thickness was $10 \mu \mathrm{m}$. Zpr1, formerly called Fret 43 [antibody stock center at The University of Oregon, (Westerfield, 1995)], was used at a 1:20 dilution overnight at $4^{\circ} \mathrm{C}$ or for $2-3 \mathrm{hr}$ at room temperature. The secondary antibody, goat anti-mouse IgG peroxidase conjugate (Sigma), was used at a 1:200 dilution for $2 \mathrm{hr}$ at room temperature.

Linkage analysis. The primers used to identify the polymorphism 
a
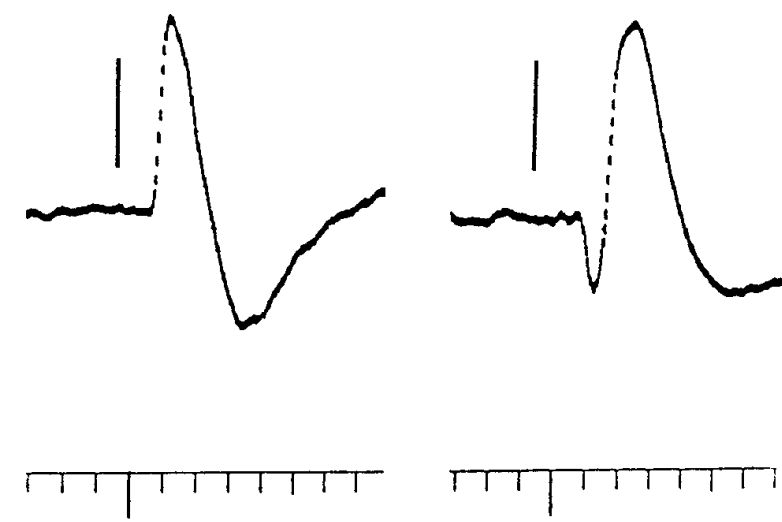

c

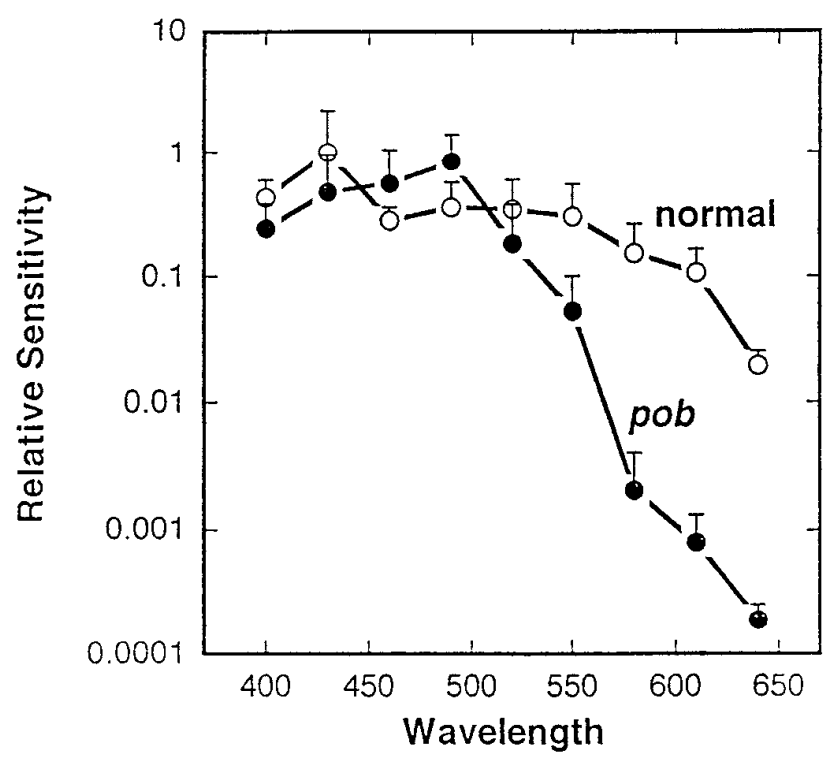

Figure 2. $a, b$, Representative ERGs from 6 dpf pob and sibling responder larvae elicited with short flashes $(10 \mathrm{msec})$ of green $(520 \mathrm{~nm})$ light at the same intensity. $a$, Responder ERG. In this recording only the $\mathrm{b}$-wave is evident. $b$, pob ERG. Both an a- and b-wave are obvious in the record. The vertical bars equal $50 \mu \mathrm{V}(a)$ or $100 \mu \mathrm{V}(b)$. Time markers $=$ $0.1 \mathrm{sec}$. The longer downward deflection along the axis indicates the flash presentation. $c$, A comparison of the spectral sensitivities of $p o b$ and sibling responder larval ERGs. Spectral sensitivities were determined by ERG analyses of 5-6 dpf pob and normal sibling larvae. The inverse of the number of photons required to generate a threshold $(20 \mu \mathrm{V})$ b-wave response was calculated at each wavelength. All data were normalized to the sensitivity of responder larvae at $430 \mathrm{~nm}$ (threshold response $=4.5 \times$ $10^{10}$ photons $\left.\cdot \mathrm{cm}^{2} \cdot \mathrm{sec}\right)$.

were "2F" (5'-ATTGGATCTTGGTCAACCTT-3') and "4R" (5'TTCCACTGAAGACATCAGGG-3'), corresponding to nucleotides 257-276 and 591-610 of the zebrafish red opsin cDNA. Two methods were used to assess linkage. In the first method primers $2 \mathrm{~F}$ and $4 \mathrm{R}$ were used to amplify the red opsin gene. After exonuclease I and shrimp alkaline phosphatase treatment, the PCR product was sequenced using a labeled dideoxynucleotide method (according to the manufacturer's instructions for "Thermo Sequenase radiolabeled terminator cycle sequencing kit"; Amersham, Arlington Heights, IL) (Fig. 6b). In the second method we used two primers [5'-AAGTTTGATGCTAAATGGGA(T/A)-3'] specific for the two red opsin alleles with the variable nucleotide at the $3^{\prime}$ end of the primer. These primers selectively amplified each allele when used in PCR in conjunction with a downstream primer. Both methods gave identical results. Ekwill-AB hybrid pob heterozygotes were used for the linkage analysis. No additional polymorphisms were detected in this strain than were detected in wild-type $\mathrm{AB}$ fish.

\section{RESULTS \\ OKR testing}

We conducted a three-generation screen for recessive mutations selectively affecting red color pathways. The strategy for generating the mutagenized fish has been used recently in large-scale screens aimed at isolating morphological mutants (Mullins et al., 1994; Solnica-Krezel et al., 1994). To identify mutants that are selectively red-blind, we illuminated the rotating stripes in our behavioral assay with red light $(>600 \mathrm{~nm})$ and then white light. Our rationale was that a red-blind mutant would not respond in red light but would respond in white light, whereas mutants with wavelength-independent defects would not respond in either red or white light. We measured the OKR of $\sim 11,000$ individual 5-6 dpf zebrafish larvae representing 198 mutagenized genomes (see Materials and Methods). One mutation ( $p o b$ ) that specifically caused red-blindness was isolated. Three morphologically normal mutants with wavelength-independent vision defects were also isolated; these will be described elsewhere.

When the rotating stripes were illuminated with red light, $\sim 25 \%$ of larvae from crosses between adults heterozygous for the pob mutation did not respond, indicating that the mutation is recessive. In white light, up to $100 \%$ of these larvae responded reliably. In contrast, $>95 \%$ of wild-type larvae responded in both red and white light with robust eye movements. pob larvae have somewhat lighter pigmentation than their normal siblings after they have been kept in dim white light or in the dark for $>10 \mathrm{~min}$ (Fig. 1). Melanophores in pob larvae do not seem to change in size in response to light, unlike melanophores of normal larvae, which contract in bright white light and expand in dim white light. Otherwise the mutant appears normal. The overall size and morphology of pob and sibling responder larvae are similar, and no morphological defects in the brain or eye were detected by external examination using a high-power dissecting microscope. Although pob larvae swim and seem morphologically normal, they die by $10 \mathrm{dpf}$. We do not yet know the cause of this lethality, but it is likely that they die from starvation, because unlike normal larvae, which begin eating at $5 \mathrm{dpf}$, $p o b$ larvae do not actively forage for food. Both $p o b$ and normal larvae were not fed in the experiments reported here.

\section{pob larvae have a defect in the outer retina}

The selective loss of red light-induced OKRs suggests that redsensitive pathways are defective in the pob mutant. To determine whether the defect is in the retina, we measured the electroretinogram elicited with both white and monochromatic light, between 400 and $640 \mathrm{~nm}$, in 5-6 dpf pob and normal sibling responder larvae. The zebrafish ERG resembles that of other vertebrates, consisting of an initial corneal negative wave (a-wave) that arises from the photoreceptors, and a larger corneal positive wave (b-wave) that arises from cells downstream to the photoreceptor cells (for review, see Dowling, 1987). The a-wave of responder larvae was usually difficult to detect, whereas the a-wave of mutant larvae was often larger than normal (Fig. 2a,b). Furthermore, the b-wave latency and time to peak were delayed in the mutant. In dim white light ( $\sim 30$ lux $)$, the latency (time from flash onset to the first $10 \mu \mathrm{V}$ positive voltage deflection) was $108 \pm 22$ $\operatorname{msec}(n=4)$ for $p o b$ mutant larvae and $58 \pm 21 \mathrm{msec}(n=9)$ for normal siblings. The time from the flash onset to the $b$-wave peak was $183 \pm 36 \mathrm{msec}(n=8)$ for pob mutants and $129 \pm 14 \mathrm{msec}$ 

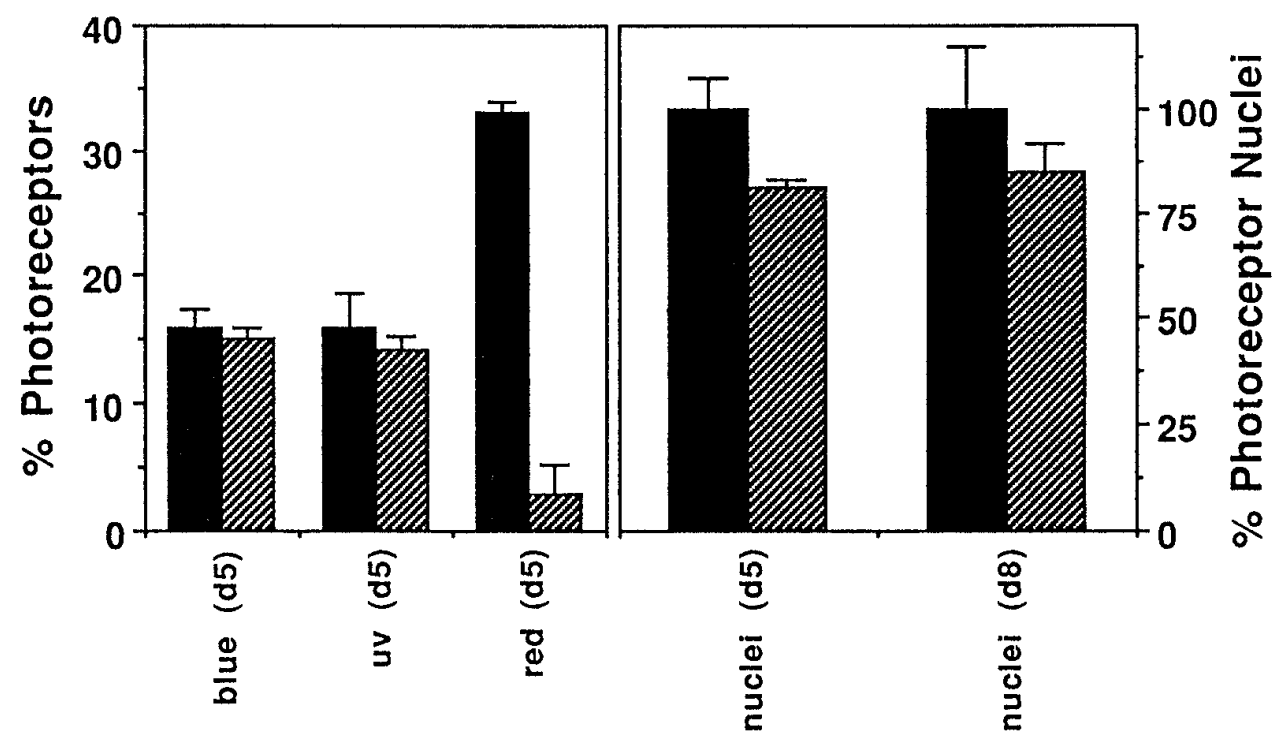

Figure 3. Left panel, A comparison of number of photoreceptors in normal sibling responder and $p o b$ retinas expressing either blue, UV, or red opsin mRNA. $p o b$ retinas have normal numbers of blue and UV cones but few red cones. Right panel, A comparison of photoreceptor nuclei number in normal sibling responder and $p o b$ retinas at $5 \mathrm{dpf}$ and at $8 \mathrm{dpf}$. pob retinas have $\sim 20 \%$ fewer nuclei in the outer nuclear layer than responder retinas.
( $n=9$ ) for normal siblings. One possibility is that the larger a-wave detected in the mutant is a secondary consequence of a delayed b-wave.

In addition, the ERG of $p o b$ larvae was significantly less sensitive to red light than was the ERG of wild-type larvae. We quantitated this difference by recording the ERG with different wavelengths of light and determining the light intensity required to elicit a threshold $(20 \mu \mathrm{V}) \mathrm{b}$-wave response. Figure $2 c$ shows relative b-wave sensitivities of pob mutants and their normal siblings between 400 and $640 \mathrm{~nm}$. Normal and pob larvae were equally sensitive to blue and green light (400-520 nm), but the sensitivity of pob mutants to light of longer wavelengths was significantly reduced. At $640 \mathrm{~nm}$, pob mutant retinas were two orders of magnitude less sensitive than their normal siblings. The selective loss of red sensitivity found in the ERG indicates that the defect in pob mutants is localized to the outer retina.

\section{pob larvae have fewer nuclei in the outer nuclear layer of the retina}

Histological analyses of pob and sibling normal responder larvae at $5 \mathrm{dpf}$ showed that the photoreceptor layer is abnormally thin in pob larvae, whereas the other retinal layers are normal (Figs. 4, 5). Quantitative studies using DAPI staining indicated that pob larvae have $\sim 20 \%$ fewer nuclei in the outer nuclear (photoreceptor) layer than do responder larvae (Fig. 3). Because twice as many red and green cones as blue and UV cones are present in the zebrafish retina and at $5 \mathrm{dpf}$ the zebrafish retina is cone dominated (Branchek and Bremiller, 1984; Raymond et al., 1995), a 20\% reduction in the number of photoreceptor nuclei suggests that a specific cone type is missing in $p o b$ retinas.

\section{pob larvae lack cones expressing red opsin}

To determine which cone type is missing, we examined opsin expression by in situ hybridization in $p o b$ and sibling responder embryos at $5 \mathrm{dpf}$ using probes corresponding to the opsins expressed in blue-, red- and UV-sensitive cones. Figure 4 shows the results obtained with blue $(a, b)$ and red $(c, d)$ opsin probes on normal $(a, c)$ and pob $(b, d)$ retinas. The results of these analyses are summarized in Figure 3. Expression of UV and blue opsins was not reduced significantly in $p o b$ embryos. In contrast, only a few photoreceptors expressing red opsin were detected in the $p o b$ retina, and these were observed primarily at the retinal margins (arrow in Fig. 4d). Because the gene encoding green opsin in zebrafish has not been isolated, we looked for the presence of green cones in pob and normal retinas using the monoclonal antibody zpr1, which recognizes an uncharacterized cell surface antigen present specifically on both red and green cones (Larison and Bremiller, 1990). Cones immunolabeled with zpr1 were detected throughout the retina of pob larvae, but fewer photoreceptors stained with zpr1 in pob than in responder retinas (Fig. 5a,b). This is consistent with the presence of green- but not red-sensitive cones in the pob retina.

\section{Red cones form and then disappear in pob larvae}

To determine whether red cones are present earlier than $5 \mathrm{dpf}$ in pob larvae, we examined red opsin mRNA expression by in situ hybridization at $3 \mathrm{dpf}$ in larvae collected from several crosses between $p o b$ heterozygotes. Because most wild-type larvae do not display a consistent OKR at $3 \mathrm{dpf}$, it was necessary to examine unsorted embryos for red opsin expression. We analyzed 70 larvae between 72 and $80 \mathrm{hr}$ postfertilization in three separate experiments. Approximately $25 \%$ of these larvae should have been mutant; however, all of these larvae expressed red opsin in photoreceptors distributed throughout the outer nuclear layer. This indicates that photoreceptors in both the mutant and wild-type retinas express red opsin at $3 \mathrm{dpf}$. These data suggest, therefore, that red cones begin to differentiate in pob but subsequently degenerate. Indeed, histological analyses of $p o b$ retinas at $5 \mathrm{dpf}$ sometimes showed evidence of cell death. Large vacuolar structures, commonly found in regions where cells have died, were occasionally seen in the photoreceptor layer of the mutant retina but were not detected in the responder retina (not shown).

The specificity of the phenotype to red cones was analyzed further by examining retinas at $8 \mathrm{dpf}$. At this stage the number of photoreceptor nuclei in pob larvae was still reduced only by $20 \%$ (Fig. 3). Furthermore, histology of the $8 \mathrm{dpf} p o b$ retina revealed no evidence of loss of additional cones (not shown). These data suggest that other cones are not degenerating at $8 \mathrm{dpf}$.

\section{The pob mutation is not in the red opsin gene}

To test whether the defect in $p o b$ retinas is attributable to a mutation in the red opsin gene, linkage between red opsin and the $p o b$ phenotype was assessed. First, a polymorphism was identified 

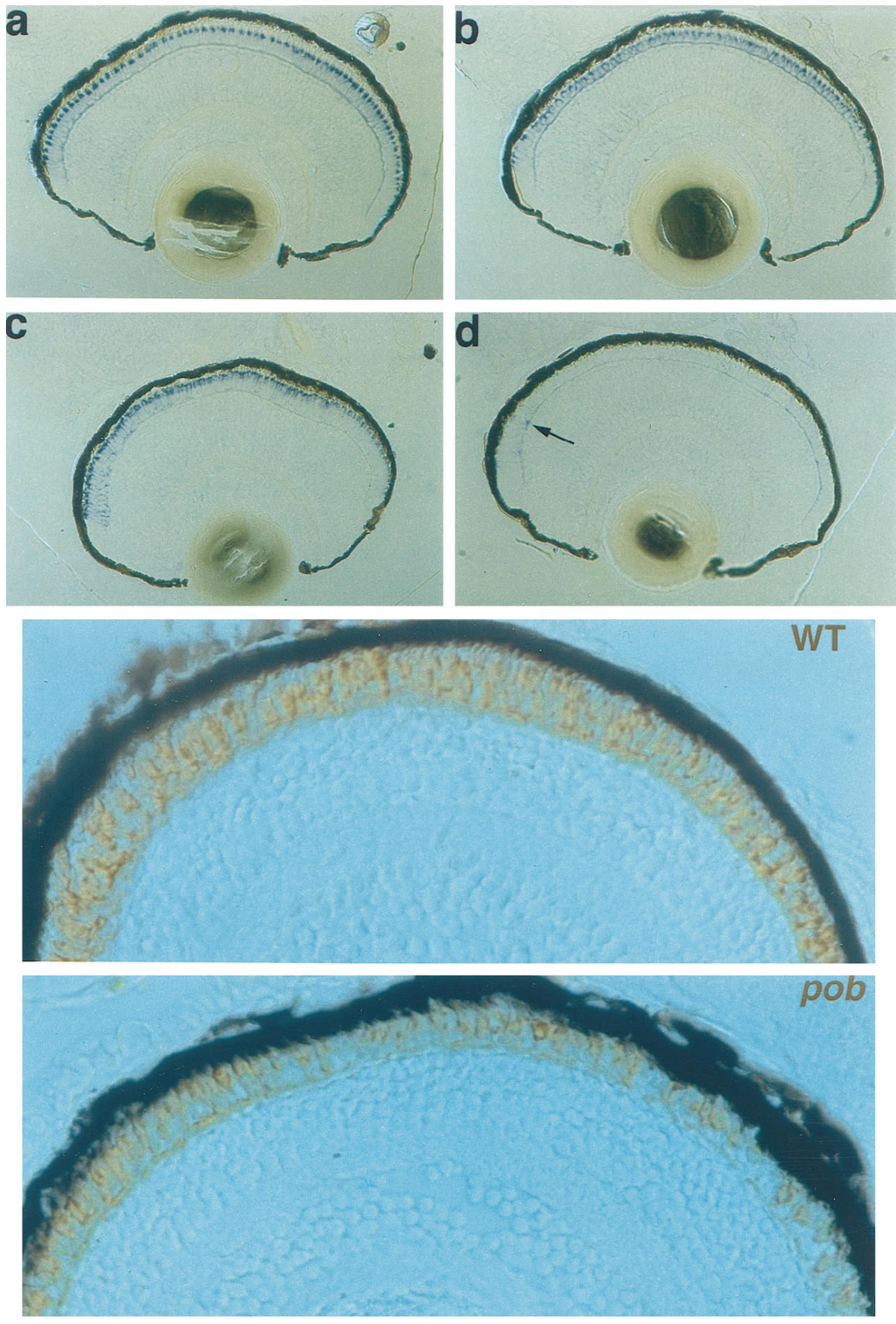

Figure 4. (Top) In situ hybridization analyses of normal (left) and pob (right) larvae at 5 dpf. $a$, $b$, Blue opsin mRNA expression; $c$, $d$, red opsin mRNA expression (the arrow points to a cell near the retinal margin expressing some red opsin mRNA).

Figure 5. (Bottom) Immunohistochemical staining of larval retinas at $5 \mathrm{dpf}$ with the monoclonal antibody zpr1. $a$, Normal sibling larva; $b$, pob larva. The retinal staining in the pob larva is likely caused by green cones.

in the red opsin gene in wild-type fish. To identify a polymorphism, we isolated and analyzed DNA from individual larvae produced from crosses between adult wild-type AB zebrafish. A portion of the red opsin gene expected to contain one or two introns based on homology with other opsin genes was amplified and sequenced. Two introns were found in the amplified PCR product, one between nucleotides 155 and 156 and the other between nucleotides 556 and 557 of the cDNA coding sequence. A naturally occurring polymorphism (either $\mathrm{A}$ or $\mathrm{T}$ ) in the $\mathrm{AB}$ population was identified at cDNA nucleotide 498 . This missense mutation converts codon GCA into GCT, both of which encode alanine (Fig. 6a). This polymorphism served as a linkage marker.

To assess linkage, two crosses between a heterozygous pob male and a heterozygous $p o b$ female were conducted. The resulting homozygous pob mutant larvae and normal responder larvae were identified by their abilities to detect either only white but not red light (NR) or both red and white light (responder) in the OKR assay. Both adult fish used for these two crosses were heterozygous for the red opsin polymorphism. Total nucleic acid was isolated from each individual mutant larva and from each indi- 


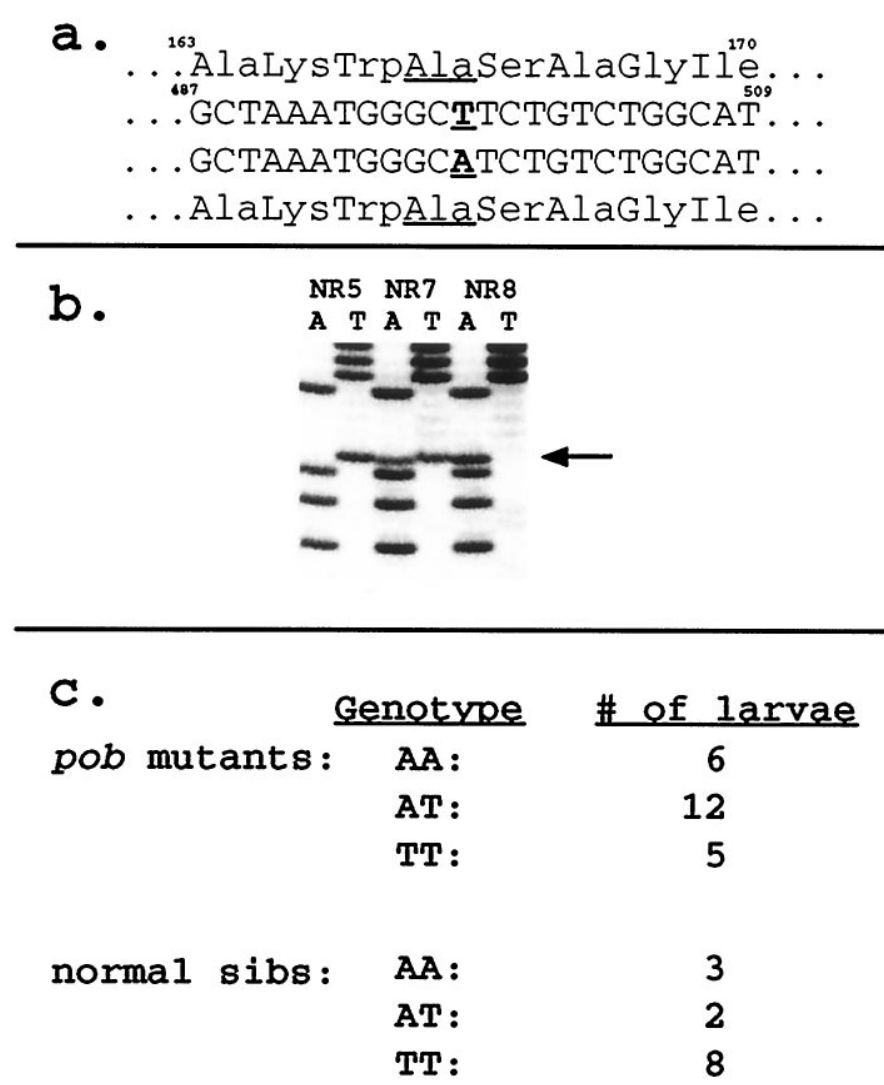

Figure 6. $a$, Amino acid and nucleotide sequences of the two polymorphic alleles of the red opsin gene found in the wild-type AB zebrafish population. Both alleles encode identical amino acid sequences. Numbering begins at the initiator methionine codon of the red opsin cDNA sequence. $b$, Sequence data (only $A$ and $T$ lanes) from reverse complement of the sequence shown in $a$ for homozygous pob mutant larvae $(N R)$ showing three different red opsin genotypes. $c$, Distribution of red opsin alleles in the progeny from two pob heterozygotes. These data indicate that the red opsin gene is not linked to the $p o b$ phenotype.

vidual normal sibling, and a portion of the red opsin gene was amplified by PCR. The products were sequenced directly as shown in Figure $6 b$. Figure $6 c$ shows that the two alleles were present in pob mutant larvae in the ratio expected if there is no linkage between red opsin and the $p o b$ phenotype; the $p o b$ phenotype and the polymorphism in red opsin segregated independently. This finding demonstrates that the defect underlying the pob mutation is not in the red opsin gene.

\section{DISCUSSION}

In this paper we describe the isolation and characterization of a recessive mutation in zebrafish that leads to the selective loss of red opsin-expressing retinal photoreceptors between 3 and $5 \mathrm{dpf}$. Methods for identifying genes responsible for mutant phenotypes are not as advanced for zebrafish as they are for other eukaryotic organisms such as Drosophila, Caenorhabditis elegans, or yeast. Nevertheless, a naturally occurring polymorphism within the wildtype $\mathrm{AB}$ strain did allow us to evaluate the most obvious candidate for the $p o b$ gene, the red opsin gene. Our analysis (Fig. 6) showed that red opsin and the $p o b$ phenotype are not linked. We conclude, therefore, that the mutation must be in another gene that is required for the maintenance or function of red-sensitive photoreceptors.

Specific color-vision defects attributable to alterations in genes other than visual pigment genes have not been described. The most common forms of color blindness, red- or green-defective dichromacy, and blue monochromacy result from abnormalities in the red and green opsin genes (Nathans et al., 1986, 1989, 1993). Tritanopia, a less common type of color blindness leading to selective loss of blue sensitivity, is caused by mutations in the blue opsin gene (Weitz et al., 1992a,b). Other molecules have not been implicated in color-specific defects, perhaps because few unique molecules besides opsins are present in different cone types, at least in mammals. For example, most cone-specific phototransduction proteins are shared by different cone types in mammals (Hurwitz et al., 1985; Lerea et al., 1989; Lee et al., 1992; Bonigk et al., 1993; Ong et al., 1995; but see Hamilton and Hurley, 1990). The only reported cone degeneration thought to be attributable to a defect in a molecule involved in cone phototransduction, characterized in dogs, leads to degeneration of all cone types (Gropp et al., 1996). Whether different cone types in fish have distinct phototransduction molecules is unknown. Because fish red and green cones diverged from one another much longer ago than did mammalian red and green cones, more molecules may be unique to red cones in fish than in mammals.

All of our histological data indicate that only the red cones are affected in pob mutants; however, the fact that the ERG of the pob mutant at 5-6 dpf often had a larger a-wave and delayed b-wave suggests that there may be some abnormality in other cones and perhaps rods as well. It is known that in certain forms of human retinitis pigmentosa, in which the gene defect is limited to rods, the cones eventually degenerate for reasons not yet understood (for review, see Berson, 1993). Even at $8 \mathrm{dpf}$, however, we saw no histological evidence of cone degeneration in the pob mutant other than red cone degeneration.

A curious phenotype observed in some of the retinal mutants we have identified is abnormal regulation of melanophore size. In $p o b$, many of the melanophores are chronically contracted. In another retinal mutant, noa, in which there appears to be a failure of synaptic transmission, the melanophores are chronically expanded (Brockerhoff et al., 1995). The pigmentation of melanophores is controlled both by neural input to the cells and by circulating neural hormones (Ujii and Novales, 1968; Bagnara, 1972). Melanin granules in zebrafish melanophores aggregate in the light and disperse in the dark, causing darkening of zebrafish at night and blanching during the day. One of the neural hormones involved in melanin aggregation is melatonin (Sugden and Rowe, 1992; Filadelfi and Castrucci, 1994). In mammals and lower vertebrates, the synthesis of melatonin occurs in both the retina and the pineal gland (Grace and Besharse, 1994; Tosini and Menaker, 1996). Melatonin synthesis is circadian but also regulated by light (Cahill and Besharse, 1993; Cahill, 1996), but how light regulates melatonin release and resets circadian clocks is unknown. The melanophore defect detected in both pob and noa mutants indicates that light is unable to efficiently regulate changes in pigmentation in these animals. This may be attributable to the retinal defects we have identified, or alternatively the pineal also may be defective in these mutants. We have observed red opsin expression in the pineal of normal larval zebrafish, but are as yet uncertain about whether red opsin expression occurs in the pineal of pob mutants.

In summary, we have identified a novel form of color-blindness in zebrafish. Red opsin-expressing cones specifically disappear in pob mutants between 3 and $5 \mathrm{dpf}$. Unlike all other reported types of inherited color defects involving a specific cone type, the mutation described here is not in an opsin gene. Because red 
opsin is the only known molecule unique to red cones, this study may have identified a novel gene that is specific for red cones.

\section{REFERENCES}

Bagnara JT (1972) Chromatophores and color change; the comparative physiology of animal pigmentation. Englewood Cliffs, NJ: Prentice-Hall.

Berson EL (1993) Retinitis pigmentosa: the Friedenwald lecture. Invest Ophthalmol Vis Sci 34:1659-1676.

Bonigk W, Altenhofen W, Muller F, Dose A, Illing M, Molday RS, Kaupp UB (1993) Rod and cone photoreceptor cells express distinct genes for cGMP-gated channels. Neuron 10:865-877.

Branchek T (1984) The development of photoreceptors in the zebrafish, Brachydanio rerio. II. Function. J Comp Neurol 224:116-122.

Branchek T, Bremiller R (1984) The development of photoreceptors in the zebrafish, Brachydanio rerio. I. Structure. J Comp Neurol 224:107-115.

Brockerhoff SE, Hurley JB, Janssen-Bienhold U, Neuhauss CF, Driever W, Dowling JE (1995) A behavioral screen for isolating zebrafish mutants with visual system defects. Proc Natl Acad Sci USA 92:10545-10549.

Clark DT (1981) Visual responses in the developing zebrafish (Brachydanio rerio). In: Biology. PhD thesis, University of Oregon.

Cahill GM (1996) Circadian regulation of melatonin production in cultured zebrafish pineal and retina. Brain Res 708:177-181.

Cahill GM, Besharse JC (1993) Circadian clock functions localized in Xenopus retinal photoreceptors. Neuron 10:573-577.

Dowling JE (1987) The retina: an approachable part of the brain. Cambridge: Belknap Press of Harvard UP.

Filadelfi AM, Castrucci AM (1994) Melatonin desensitizing effects on the in vitro responses to $\mathrm{MCH}$, alpha-MSH, isoproterenol and melatonin in pigment cells of a fish (S. marmoratus), a toad (B. ictericus), a frog (R. pipiens), and a lizard (A. carolinensis), exposed to varying photoperiodic regimens. Comp Biochem Physiol [A] Physiol 109:1027-1037.

Grace MS, Besharse JC (1994) Melatonin deacetylase activity in the pineal gland and brain of the lizards Anolis carolinensis and Sceloporus jarrovi. Neuroscience 62:615-623.

Gropp KE, Agoston S, Huang JC, Acland GM, Farber DB, Aguirre GD (1996) Selective absence of cone outer segment $\beta_{3}$-transducin immunoreactivity in hereditary cone degeneration (cd). Exp Eye Res 63:285-296.

Hamilton SE, Hurley JB (1990) A phosphodiesterase inhibitor specific to a subset of bovine retinal cones. J Biol Chem 265:11259-11264.

Hurwitz RL, Bunt-Milam AH, Chang ML, Beavo JA (1985) cGMP phosphodiesterase in rod and cone outer segments of the retina. J Biol Chem 260:568-573.

Larison K, Bremiller R (1990) Early onset of phenotype and cell patterning in the embryonic zebrafish retina. Development 109:567-576.

Lee RH, Lieberman BS, Yamane HK, Bok D, Fung BK (1992) A third form of the $G$ protein beta subunit. 1. Immunochemical identification and localization to cone photoreceptors. J Biol Chem 267:24776-24781.

Lerea CL, Bunt-Milam AH, Hurley JB (1989) Alpha transducin is present in blue-, green-, and red-sensitive cone photoreceptors in the human retina. Neuron 3:367-376.

Mullins MC, Hammerschmidt M, Haffter P, Nusslein-Volhard C (1994) Large-scale mutagenesis in the zebrafish: in search of genes controlling development in a vertebrate. Curr Biol 4:189-202.
Nathans J, Piantanida TP, Eddy RL, Shows TB, Hogness DS (1986) Molecular genetics of inherited variation in human color vision. Science 232:203-210.

Nathans J, Davenport CM, Maumenee IH, Lewis RA, Hejtmancik JF, Litt M, Lovrien E, Weleber R, Bachynski B, Zwas F, Klingaman R, Fishman G (1989) Molecular genetics of human blue cone monochromacy. Science 245:831-838.

Nathans J, Maumenee IH, Zrenner E, Sadowski B, Sharpe LT, Lewis RA, Hansen E, Rosenberg T, Schwartz M, Heckenlively JR, Traboulsi E, Klingaman R, Bech-Hunsen NT, LaRoche GR, Pagon RA, Murphey WH, Weleber RG (1993) Genetic heterogeneity among blue-cone monochromats. Am J Hum Genet 53:987-1000.

Ong OC, Yamane HK, Phan KB, Fong HK, Bok D, Lee RH, Fung BK (1995) Molecular cloning and characterization of the $G$ protein gamma subunit of cone photoreceptors. J Biol Chem 270:8495-8500.

Oxtoby E, Jowett T (1993) Cloning of the zebrafish krox-20 gene (krx-20) and its expression during hindbrain development. Nucleic Acids Res 21:1087-1095.

Raymond PA, Barthel LK, Curran GA (1995) Developmental patterning of rod and cone photoreceptors in embryonic zebrafish. J Comp Neurol 359:537-550.

Raymond PA, Barthel LK, Stenkamp DL (1996) The zebrafish ultraviolet cone opsin reported previously is expressed in rods. Invest Ophthalmol Vis Sci 37:948-950.

Reece RJ, Robinson J, Dowling JE (1993) Zebrafish (Brachydanio rerio) visual pigments: sequence and localization. Invest Ophthalmol Vis Sci [Suppl] 35:1710.

Robinson J, Schmitt EA, Harosi FI, Reece RJ, Dowling JE (1993) Zebrafish ultraviolet visual pigment: absorption spectrum, sequence, and localization. Proc Natl Acad Sci USA 90:6009-6012.

Schmitt EA, Dowling JE (1994) Early eye morphogenesis in the zebrafish, Brachydanio rerio. J Comp Neurol 344:532-542.

Solnica-Krezel L, Schier AF, Driever W (1994) Efficient recovery of ENU-induced mutations from the zebrafish germline. Genetics 136:1401-1420.

Stenkamp DL, Hisatomi O, Barthel LK, Tokunaga F, Raymond PA (1996) Temporal expression of rod and cone opsins in embryonic goldfish retina predicts the spatial organization of the cone mosaic. Invest Ophthalmol Vis Sci 37:363-376.

Sugden D, Rowe SJ (1992) Protein kinase C activation antagonizes melatonin-induced pigment aggregation in Xenopus laevis melanophores. J Cell Biol 119:1515-21.

Tosini G, Menaker M (1996) Circadian rhythms in cultured mammalian retina. Science 272:419-421.

Ujii R, Novales R (1968) Tetrodotoxin: effects on fish and frog melanophores. Science 160:1123-1124.

Weitz CJ, Miyake Y, Shinzato K, Montag E, Zrenner E, Went LN, Nathans J (1992a) Human tritanopia associated with two amino acid substitutions in the blue-sensitive opsin. Am J Hum Genet 50:498-507.

Weitz CJ, Went LN, Nathans J (1992b) Human tritanopia associated with a third amino acid substitution in the blue-sensitive visual pigment. Am J Hum Genet 51:444-446.

Westerfield M (1995) The zebrafish book: a guide for the laboratory use of zebrafish (Brachydanio rerio). Eugene, OR: University of Oregon. 